Special Issue on KEER 2012

ORIGINAL ARTICLE

\title{
Multimodal Evaluation of Stress Level Affected by Beverage Intakes
}

\author{
Tsuyoshi MORIYAMA*, Ai YOTO** and Hidehiko YOKOGOSHI** \\ * Department of Media and Image Technology, Tokyo Polytechnic University, 1583 Iiyama, Atsugi, Kanagawa 243-0297, Japan \\ ** School of Food and Nutritional Sciences, University of Shizuoka, 52-1 Yada, Suruga-ku, Shizuoka 422-8526, Japan
}

\begin{abstract}
We propose an automated image analysis of facial expressions for measuring interventional effect of diet with respect to the mental aspect. Facial expression analysis that is noninvasive and noncontact with human subjects is promising in the sense that it can measure our natural behaviors due to emotional and biological states safely with low cost for lengthy period of time. This paper shows the analyses on the comparison between before and after the subjects ingest test samples, and also between effects of different test samples with respect to both the outputs from conventional sensors and image analyses of facial muscle activities. The subjects in our experiment (4 young adults, 2 males and 2 females) participated in a randomized and crossed over designed study for two separated experiment days. Water and green tea $(250 \mathrm{ml}$, respectively) were used as test samples. The measurements were recorded 1) before the ingestion, 2) 30 minutes after the ingestion, and 3) 60 minutes after the ingestion. During the time between each measurement, the subjects were asked to take an arithmetic mental task (AT) for 20 minutes as a stressor. Our results suggest a possibility that automated image analysis of facial expressions is one of the useful tools for evaluating the changes on mood and stress level affected by foods.
\end{abstract}

Keywords: Facial expression, Image analysis, Stress level, Beverage intakes

\section{INTRODUCTION}

Taking an immediate action on the mental care for people is an urgent demand for present urban societies. Major interventions for health care include exercise, rest, and diet. Our work focuses on the interventional effect of diet with respect to the mental aspect. Conventional measures for that purpose include electrophysiological study (e.g., electroencephalograms (EEG)) and psychological evaluations (e.g., profiles of mood states (POMS) and visual analog scale (VAS)). Although both of them are useful and trustworthy, the former requires the subjects to wear electrodes attached to their skin and is also sensitive to other stimuli than the dietary input in question and the latter requiring filling in questionnaire repetitively across lengthy period of time is not always feasible. Better means that do not require the subjects any efforts or conditions are needed for analyzing spontaneous and natural human behaviors, which lead to preventive care and commercial research.

Facial expressions, in the meantime, are known to reflect both physiological and mental activities of humans. Passively taking images of facial expressions using camera and parameterizing them from time varying changes of the image appearance enable a noninvasive and noncontact mean for inferring both physiological and mental activities of the person. It is safe, costless, and suitable for use for lengthy period of time.

We propose an automated image analysis of facial expressions for parameterizing mood of a person. In the experimental setting that we conducted in this paper, only mood that was related to stress level of the subjects was of interest. They were asked to ingest a couple of beverages (test samples) one of which was supposed to lower the stress level. Given a stressor at the beginning of each session, both the outputs conventional sensors and image analysis of facial muscle activities were compared 1) before the ingestion, 2) 30 minutes after the ingestion, and 3) 60 minutes after the ingestion. The strong correlation between them of the result demonstrates facial expressions and their analyses can be an alternative measurement for analyzing human moods and be applied to the mean for mental care.

\section{MENTAL STATES AND HUMAN BEHAVIORS}

Human behaviors are affected by both the mental and the physiological activities. They include internal and invisible activities (e.g., neural response in the brain and emotion experienced by the subjects) and external and visible ones (e.g., facial muscle movements). The former which has conventionally been measured by using electrophysiological sensors (e.g., electroencephalograms 
(EEG)) and psychological evaluations (e.g., POMS and VAS) has been used for analyzing mood and stress level. It needs sensors attached to the subjects and makes the subjects to be aware of being measured that may affect the result. The latter, on the other hand, has been investigated for a couple of decades and analyzing facial muscle activities from face images has several advantages compared with the former, i.e., noninvasive, noncontact with the subject, low cost, and safe that allows to measure natural behaviors for a lengthy period of time. Thus, the latter is a supplementary or an alternative for the former.

\section{FACE ANALYSIS FOR MOOD DETECTION}

Human affection consists of emotions and moods. Emotions take short period of time for occurrence and the strengths tend to be strong, whereas moods take long time and the strengths and their time difference are both subtle, and more importantly, they give a strong bias to the kind of emotions likely to emerge. For example, when a person is in irritated mood, he or she tends to get angry rather than smile. From the viewpoint of mental and health care, taking moods for measuring mental and physiological states of humans would be more feasible than analyzing emotions because moods are dominant over emotions. Also, detecting moods from facial expressions would give a better observation due to the reason explained in the previous section.

\section{MOOD DETECTION BY FACE IMAGE ANALYSIS}

Our preliminary experiments show that moods categorized in negative arousal (e.g., irritated and tense) typically are accompanied with specific facial expressions. They include mouth opening (AU25 in the Facial Action Coding System (FACS) [2]), narrowing eyebrows that sometimes causes wrinkling between eyebrows (AU4, 9), widening eyes (AU1, 2, 5). Also, head motion is a strong cue when talking about attention that is supposed to be the inverse of fatigue. When people focus on something or they feel sleepy, they tend not to move their heads.

\subsection{Head motion tracking}

We use a head tracker that is based on a 3D cylindrical head model [1]. Manually given the head region with the pose and feature point locations (e.g., eye corners) in an initial frame, the tracker automatically builds the cylindrical model and recovers 3D head poses and feature point locations across the rest of the sequence. The initial frame is selected such that it has the most frontal and upright face in it. The tracker recovers full 3D rigid motions (three rotations and three translations) of the head. The performance evaluation on both synthetic and real images has demonstrated that it can track as large as 40 degrees and 75 degrees of yaw and pitch, respectively, within 3.86 degrees of average error. As shown in Figure 1, the stabilized face images cancel out most of the effect of 3D head pose and contain only the remaining non-rigid facial expression.

\subsection{Eye motion tracking}

Figure 2 shows a schematic overview of the whole process of a model-based eye region image analysis system for tracking eye motions [3]. An input image sequence contains facial behaviors of a subject. Facial behaviors usually accompany spontaneous head motions. The appearance changes of facial images thus comprise both rigid 3D head motions and non-rigid facial actions. Decoupling these two components is realized by recovering the $3 \mathrm{D}$ head pose across the image sequence and by accordingly warping the faces to a canonical head pose (frontal and upright), which we refer to as the stabilized images; Stabilized images are intended to include appearance changes due to facial expressions only. Eye image analysis proceeds on these stabilized images. For a given stabilized image sequence, the system registers the eye region model $T\left(\mathbf{x} ; \mathbf{s}, \mathbf{m}_{t}\right)$ to the input $I$ in the initial frame and individualizes the model by adjusting the structure parameters s. Motion of the eye is then tracked by estimating the motion parameters $\mathbf{m}_{t}$ across the entire image
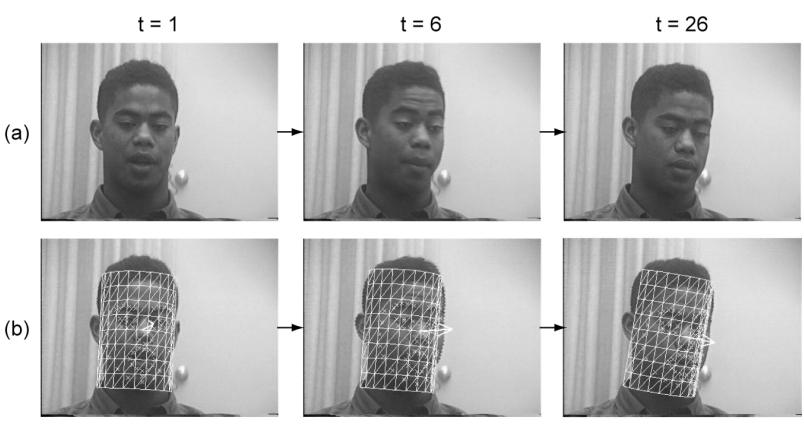

(c)

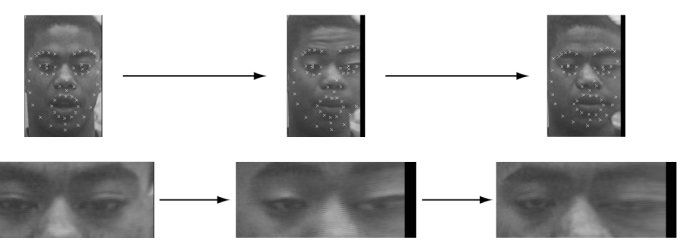

Figure 1: Automatic recovery of 3D head motion and image stabilization [1]. (a) Frames 1, 10, and 26 from original image sequence. (b) Face tracking in corresponding frames. (c) Stabilized face images. (d) Localized face regions. 


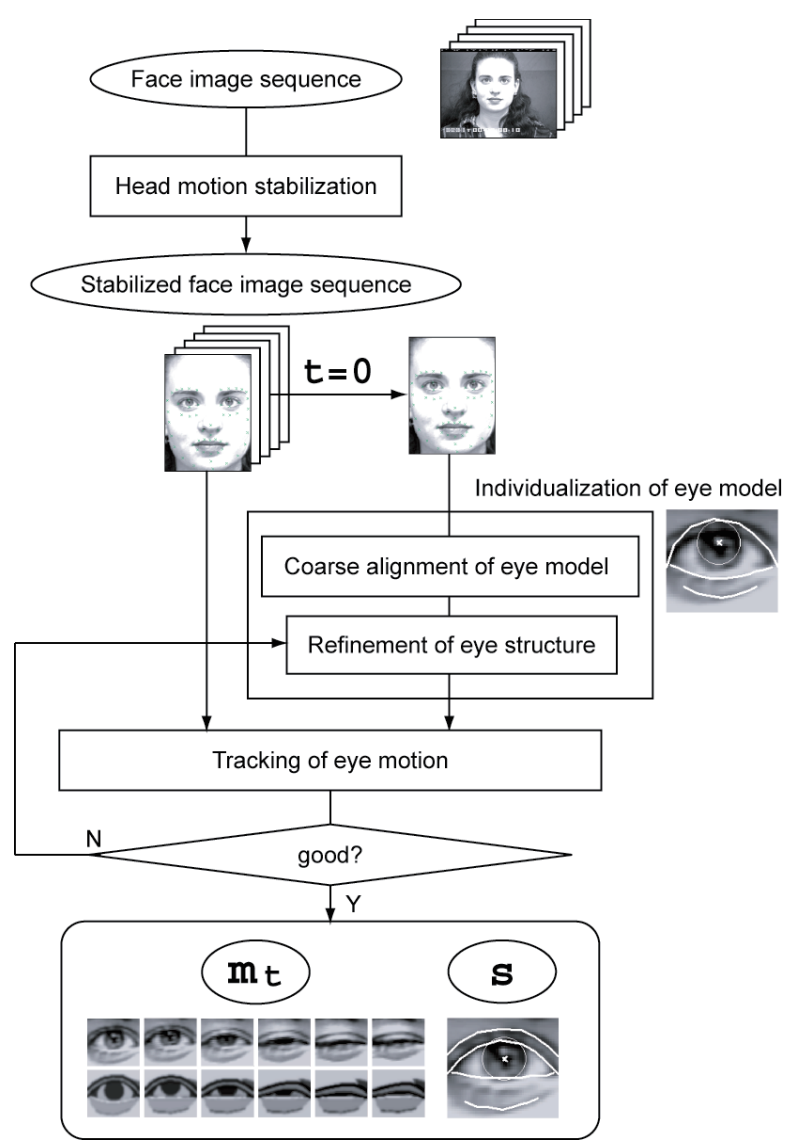

Figure 2: A schematic overview of the model based eye image analysis system.

sequence. If the tracking results at any time $t$ are off the right positions, the model is readjusted, otherwise finally get the estimated motion together with the structure of the eye. Starting with the values in the previous frame, the motion parameters $\mathbf{m}_{t}$ at the current frame $t$ are estimated by minimizing the following objective function $D$ :

$$
D=\sum\left[T\left(\mathbf{x} ; \mathbf{m}_{t}+\delta \mathbf{m}_{t}\right)-I\left(W\left(\mathbf{x} ; \mathbf{p}_{t}+\delta \mathbf{p}_{t}\right)\right)\right]^{2}
$$

where $I$ is the input eye region image, $W$ is a warp from the coordinate system of the eye region model to that of the eye region image, and $\mathbf{p}_{t}$ is a vector of the warp parameters that includes only translation in this implementation. Structure parameters $\mathbf{s}$ do not show up in $T$ because they are fixed while tracking. $\delta \mathbf{m}_{t}$ and $\delta \mathbf{p}_{t}$ are obtained by solving the simultaneous equations obtained from the first-order Taylor expansion of (1). $\mathbf{m}_{t}$ and $\mathbf{p}_{t}$ are updated:

$$
\mathbf{m}_{t} \leftarrow \mathbf{m}_{t}+\delta \mathbf{m}_{t}, \quad \mathbf{p}_{t} \leftarrow \mathbf{p}_{t}+\delta \mathbf{p}_{t}
$$

The iteration process at a particular frame $t$ converges when the absolute values of $\delta \mathbf{m}_{t}$ and $\delta \mathbf{p}_{t}$ become less than the preset thresholds or the number of iterations reaches the maximum.

\section{MATERIALS AND METHOD}

The experiment conducted in this study was approved by the research ethics committee of the University of Shizuoka and was carried out in accordance with the Declaration of Helsinki.

\subsection{Subjects}

Four subjects ( 2 males and 2 females, ages: $22.8 \pm 0.5$ years old) participated in this experiment. All subjects were healthy volunteers and right-handed, and gave written informed consent for study participation prior to their participation.

\subsection{Stimuli}

Two arithmetic tasks (10 minutes for each) and an auditory target detection task (5 minutes) were imposed as mental stress tasks. Subjects were requested to add two numbers randomly displayed on a PC monitor and type the answer in the answer column with a numerical keypad as quickly and accurately as possible. After the first arithmetic task, subjects rested for 1 minute, and then another arithmetic task was performed. In the auditory target detection task, subjects responded to target stimuli that occurred infrequently and irregularly within a series of standard stimuli (target stimuli: $2 \mathrm{kHz}$ single tones, standard stimuli: $1 \mathrm{kHz}$ single tones). Subjects were requested to click the left mouse button as quickly as possible when they heard the target stimuli. We also used two kinds of beverages, i.e., green tea and water, as the test samples for inducing different mood after stress load tasks. A cross-over, randomized design was used in this study. Two separate trials were performed in which the participants orally took either 250 $\mathrm{ml}$ of green tea or water (both at 25 Celsius) each day.

\subsection{Camera settings for taking face images}

Figure 3 shows the positions of two cameras relative to the subject. The images captured by the cameras were

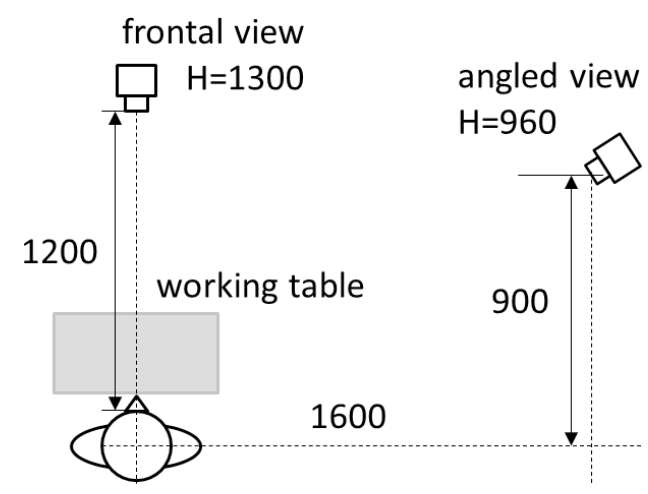

Figure 3: Camera settings. 
sent to DIGITAL HANDS VD-100 [4] with the audio signal captured by the microphone and recorded in a single disk file synchronously to each other. Cameras were set hidden so that the subjects would not notice them. An example set of recorded face images are shown in Figure 4.

\subsection{Subjective assessment}

The POMS and the VAS, used for subjective ratings on mood state, were completed before the intakes for baseline data and after the mental stress tasks were finished. The short version of POMS was held to assess distinct affective mood states. VAS comprises seven scales including the feelings of pressure, drowsiness, stress, relaxation, fatigue, arousal, stressful, reassurance and tension. The changes of VAS ratings after mental stress tasks from the baseline ratings before sample intakes were calculated to the standard $\mathrm{z}$-scores for the analysis.

\subsection{Procedure}

Each participant was required to attend a total of 2 study days for about 1 hour per day. Prior to the start of the experiment, all participants were given the opportunity to familiarize themselves with all of the mental stress tasks. Experiments took place in a quiet room with the temperature of about 25 Celsius and the relative humidity of about $50 \%$. On the experiment day, the participant entered the room, was seated and rested for 15 minutes. During the resting time, electrodes were attached. After the rest, a measurement session and subjective assessment for baseline data took place. The cameras and microphone were kept capturing the face of the subject and the vocal instructions of the experimenter's from the beginning through the end. After the sample intake, the participant performed the mental stress tasks, and then at around 30 minutes after the sample intake, another measurement session was completed. At last, the electrodes were taken off, and the participant left the experiment room.

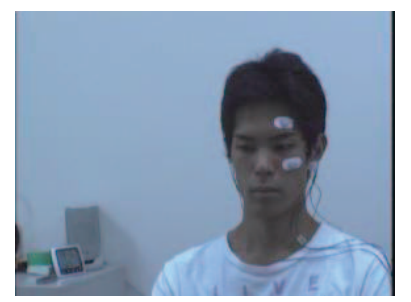

(a) frontal view

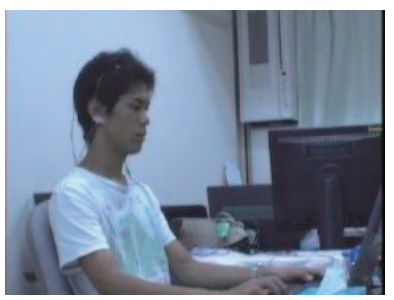

(b) angled view
Figure 4: An example set of face images.

\subsection{Statistical analysis}

The collected data were analyzed using IBM SPSS Statistics version 19. Nonparametric Mann-Whitney's U tests were performed to detect differences between the two samples (i.e., water and green tea). Pearson's correlation coefficients between the head motion tracking after each arithmetic task and the changes of VAS z-scores were also performed to evaluate their correlations.

\section{RESULTS}

\subsection{VAS}

Figure 5(a) presents the averaged changes of each of the 6 rating scores of VAS among subjects. The results of non-parametric Mann-Whitney's U tests between the two intake samples showed that the feeling of stress caused by the mental stress tasks after water sample intake was larger than that after green tea intake $(\mathrm{P}<0.05)$, and the feeling of relaxation was stronger in the green tea intake condition than that in the water condition. This result supports the fact that green tea has an effect of reducing mental stress after stress load tasks.

\subsection{Head motion tracking}

Figure 6 presents an example set of image frames and the correspondent tracking results. In Figure 6(b), the cylindrical mesh superimposed over the head region indicates the head model and the arrow on the top of nose indicates the head orientation. The cylindrical model of head was successfully matched with the input head in each frame.

Figure 5(b) shows the dynamic ranges measured after both task 1 and task 2 . The dynamic ranges in green tea intake condition tended to be smaller than that measured after task 1 significantly $(\mathrm{P}<0.1)$. Here, the head orientation is the root mean square of the 3 degrees of freedom in orientation (yaw, pitch, and roll).

\subsection{Correlation between VAS and head motion}

The results of the Pearson's correlation coefficients between the tracked head motions after arithmetic task 2 and the changes in VAS z-scores are shown in Figure 7. The correlations between the dynamic range of the head orientation and both ratings of stress and tension, and that between the dynamic range of the head translation and the ratings of drowsiness were found significant $(\mathrm{P}<0.05)$. Here, the head translation is the root mean square of the 3 degrees of freedom in translation (horizontal, vertical, and depth directions). 


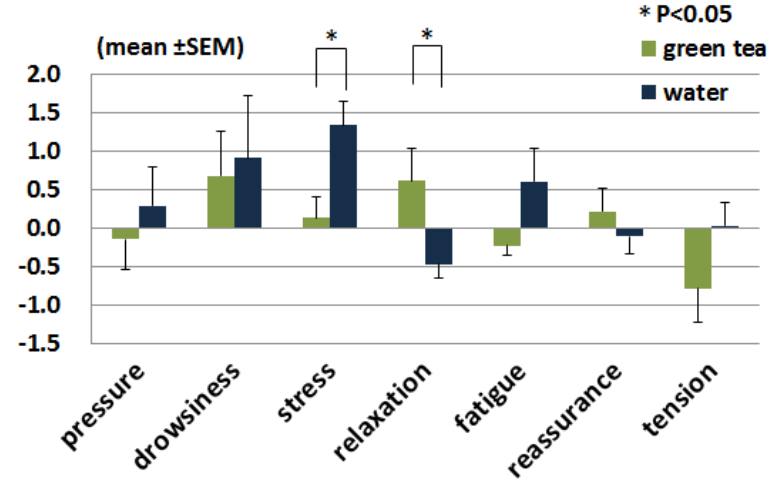

(a) Changes of VAS (z-score) in the 6 scales, i.e., feelings of pressure, drowsiness, stress, relaxation, fatigue, arousal, stressful, reassurance and tension.

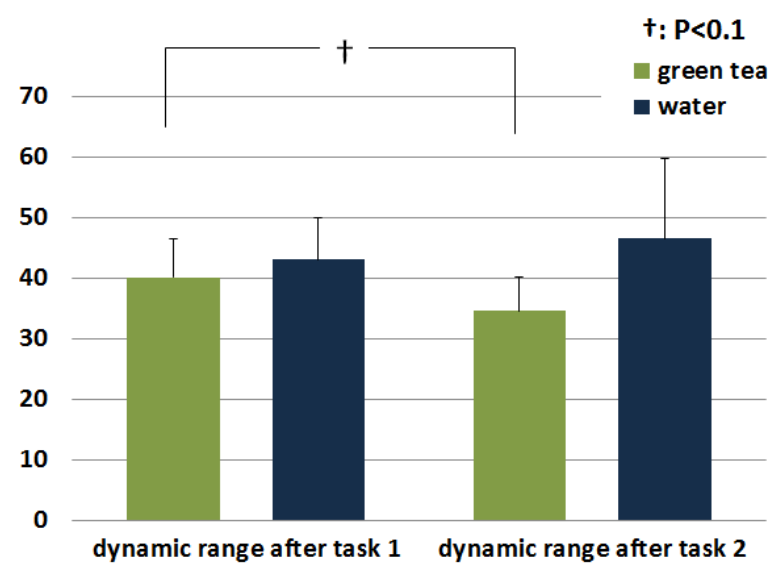

(b) Dynamic ranges of head orientation: a comparison between ones after task 1 and those after task 2 .

Figure 5: Result of comparison performed by non-parametric Mann-Whitney's U tests.

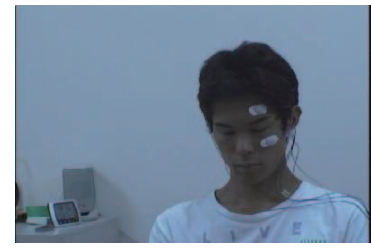

(a) input $\# 0$

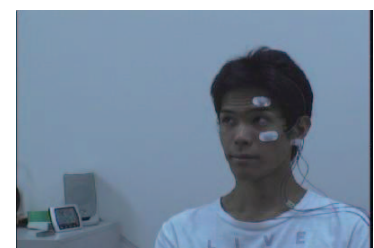

(c) input \#525

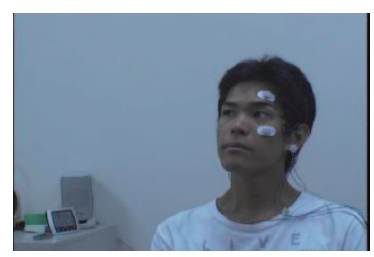

(e) input $\# 880$

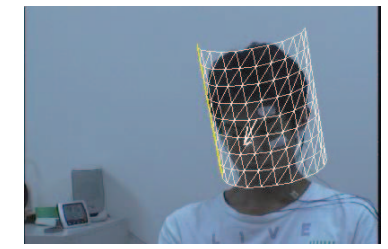

(b) tracking $\# 0$

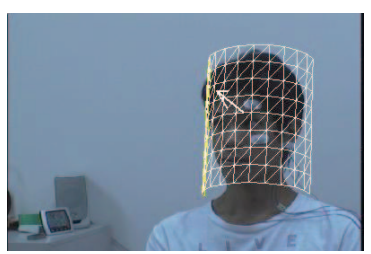

(d) tracking \#525

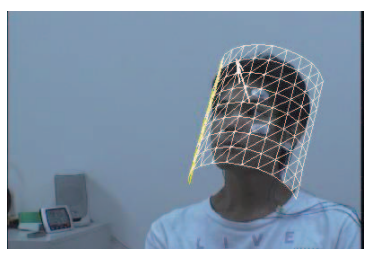

(f) tracking \#880
Figure 6: An example set of tracking results.

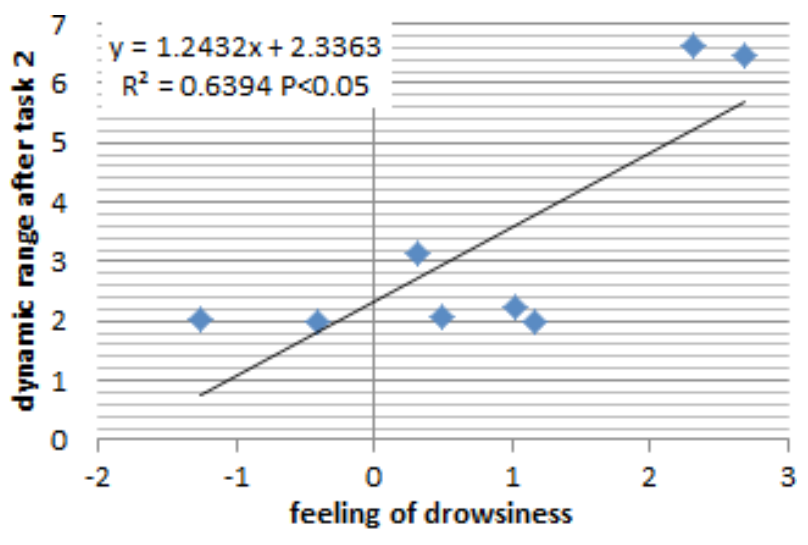

(a) Correlation between the dynamic rage of the head orientation and the stress ratings in VAS.

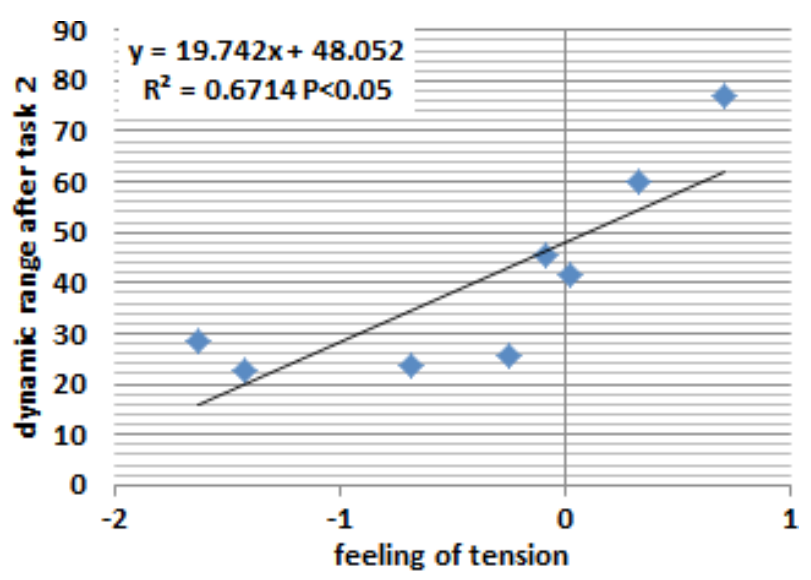

(b) Correlation between the dynamic rage of the head orientation and the tension ratings in VAS.

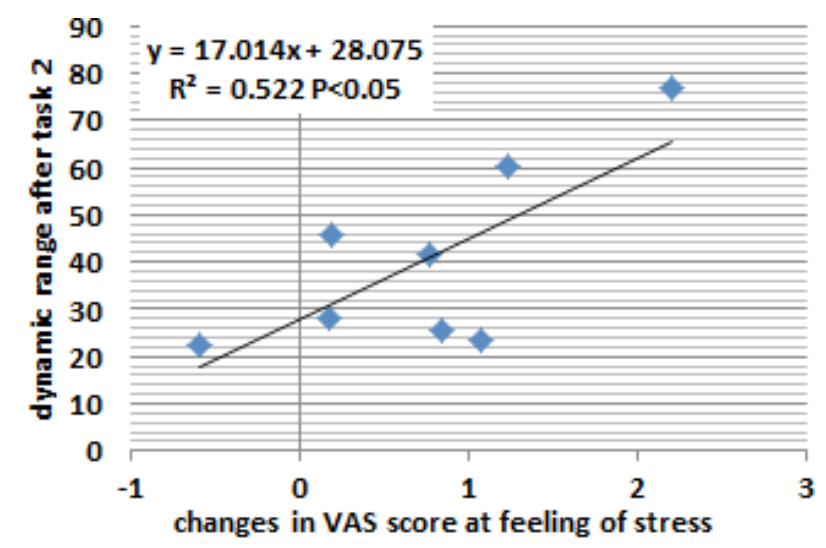

(c) Correlation between the dynamic rage of the head translation and the drowsiness ratings in VAS.

Figure 7: Correlations between the tracked head motion after the arithmetic task 2 and the changes in VAS scores. The Pearson's correlation coefficients were performed to obtain the above relationships. 


\subsection{Eyelid movements during resting time after the arithmetic tasks}

Figure 8 shows observed expressions in the eye regions for two of the subjects out of four, the rest of whom had glasses and long hair that hid their eye regions. The horizontal axes indicate time in minutes passed since the

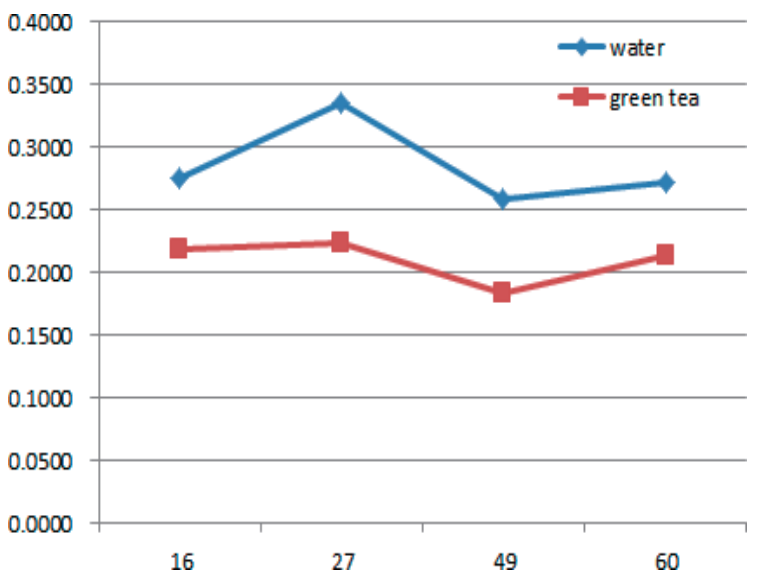

(a) Average of eye heights for Subject \#1

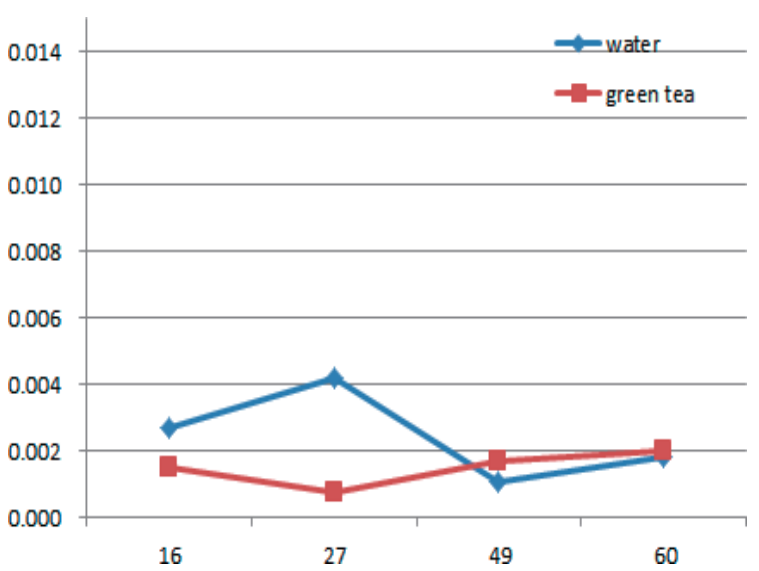

(c) Variance of eye heights for Subject \#1

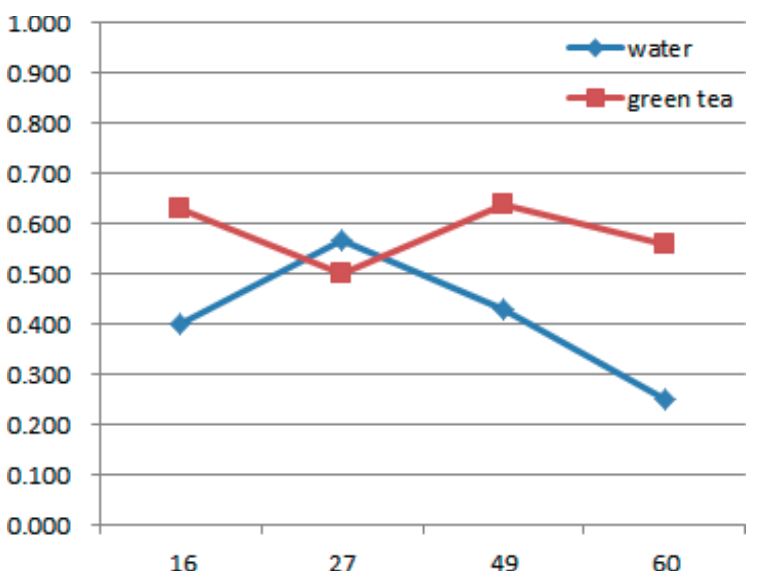

(e) Blink rate for Subject \#1

Figure 8: An example set of eye movement results. ingestion of the test samples. According to the past work $[7,8]$, the following discussion focuses only on the data after 27 minutes since the ingestion. Figure 8(a) and (b) show the average of time-varying aspect ratio of the eye aperture (the height normalized by the width) that indicates how wide the eyes were opened, Figure 8(c) and (d)

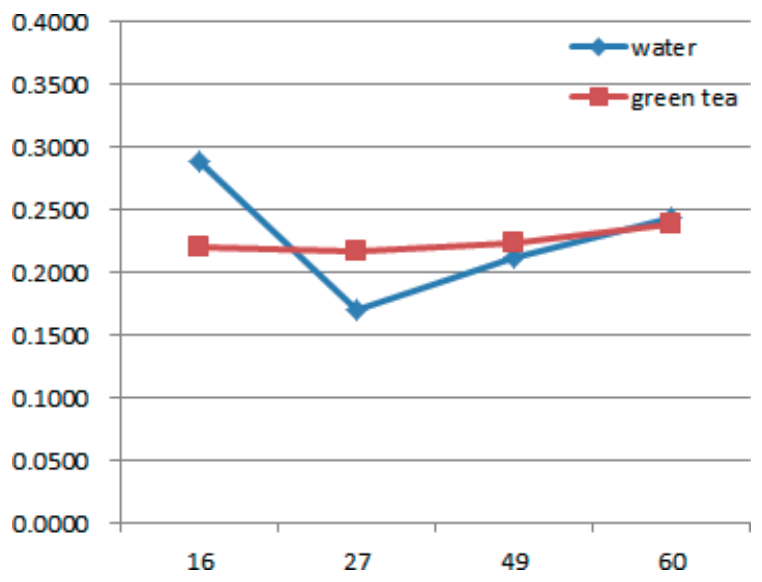

(b) Average of eye heights for Subject \#2

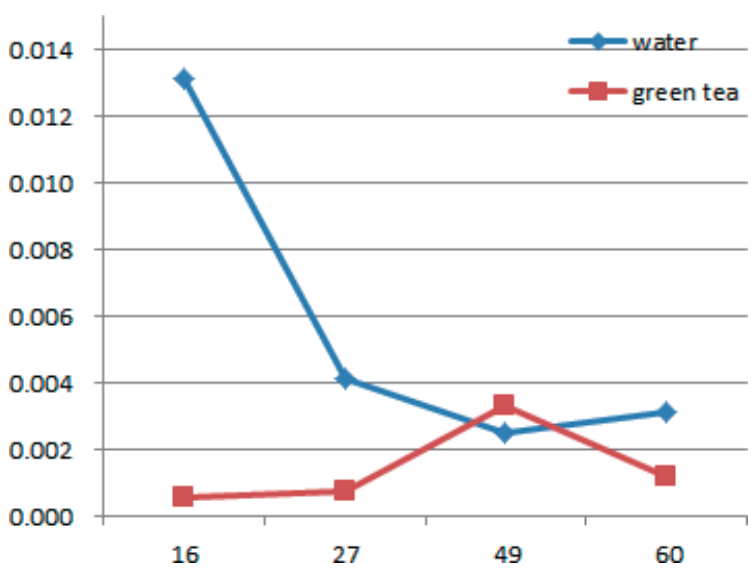

(d) Variance of eye heights for Subject \#2

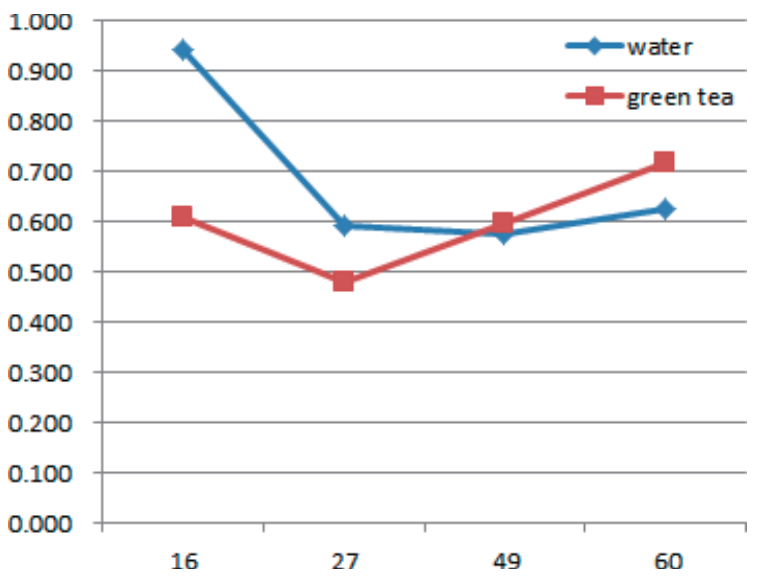

(f) Blink rate for Subject \#2 
show the variance of the time-varying aspect ratio of the eye aperture that indicates how frequently the eyes looked up and down, and Figure 8(e) and (f) show the blink rates per second that indicate how often eye blinking occurred. Due to lack of the number of subjects, the results only suggest a rough analysis of the relation between the stress level and the eye movement. As to the aspect ratio of the eye region, Figure 8(a), (b), (c), and (d) show the average for both water intake and green tea intake conditions stayed approximately constant, whereas the variance showed opposite characteristics between them, i.e., that with water got less as time passed, whereas that with green tea got larger. As to blink rate, Figure 8(e) and (f) showed that with water got less as time passed, whereas that with green tea got larger.

\section{DISCUSSION}

Figure 5(a) showed significant difference between green tea and water in the effect of reducing stress level after the arithmetic tasks. Also, Figure 5(b) showed that dynamic range of head orientation after the arithmetic task 2 decreased only with green tea (it rather increased with water). With these two evidences, Figure 7 indicates there were significant positive correlation between the dynamic range of the head orientation and both ratings of stress and tension, and that between the dynamic range of the head orientation and the ratings of drowsiness. It appears to support the hypotheses that green tea has an effect of reducing mental stress, and more importantly, image analysis of facial expressions can be an alternative measure for evaluating stress level in addition to conventional methods such as EEG.

As shown in Figure 5(b), there was no significant difference between water intake and green tea intake conditions even though there was a significant difference in the stress ratings (VAS scores). Possible reasons for this are: 1) the variance among subjects was too large compared with the number of subjects that was relatively small, and 2) the timing of evaluating anti-stress effect by the green tea intake after task 1 and task 2 may have been too early because VAS, on the other hand, was performed around 10 minutes after task 2 . In other words, the difference of the dynamic range between the two sample conditions could be detected at the timing of 10 minutes after the mental task 2 . We would make this clear in our future study.

Regarding eye movements shown in Figure 8, the possible rationale for the result of blink rate (Figure 8(e) and (f)), the stress level could have been lowered as time passed because past research reported that blink rate would decrease as mental workload increases [5]. Comparison between the result of the variance of the aspect ratio of the eye aperture (Figure 8(c) and (d)) and that of VAS (Figure 5(a)) would support the rationale that relaxing and being released from fatigue by green tea intake urged the subjects to make larger look-around behaviors as time passed, whereas the same behavior got less frequent in the case of water intake.

Past studies have reported that green tea that we used as one of the test samples contains several components that have an effect of reducing stress level under mental stress tasks, such as L-theanine (Haskell et al. 2008 [6]) and $\gamma$-aminobutyric acid (Yoto et al. 2011 [7]). We examined if image analysis of facial expressions could be an additional measure for evaluating stress level to conventional methods such as EEG. We conducted an experiment where the subjects took mental stress tasks while images of their faces being taken by camera. The experimental results demonstrated that dynamic range of head motion, which is one of facial expressions defined in FACS [2], was correlated with subject assessment of mood measured by VAS. The findings indicate that mental effects of other beverage intakes with stress tasks can be measured using image analysis of face images.

\section{CONCLUSION}

Our results suggest a possibility that automated image analysis of facial expressions is one of the useful tools for evaluating the changes on mood and stress level affected by foods. Applying the proposed method for developing anti-stress foods and beverages ultimately realizes an evaluation tool for developing many kinds of products that can prevent depression and other affection related disease. Future work includes increasing the number of the subjects for further investigating the correlation between the stress level and eye movements.

\section{ACKNOWLEDGEMENTS}

We thank all the participants who cooperated with us on the stressful experiments. This study was partly supported by a project of Shizuoka Prefecture and Shizuoka City Collaboration of Regional Entities for the Advancement of Technological Excellence, Japan Science and Technology Agency (JST). 


\section{REFERENCES}

1. Xiao, J., Moriyama, T., Kanade, T., and Cohn, J.F.; Robust Full-Motion Recovery of Head by Dynamic Templates and Re-Registration Techniques, Int'1 J. Imaging Systems and Technology, 13, pp.85-94 (2003).

2. Ekman, P. and Friesen, W.; Facial Action Coding System, Consulting Psychologists Press (1978).

3. Moriyama, T., Kanade, T., Xiao, J., and Cohn, J.F.; Meticulously Detailed Eye Region Model and Its Application to Analysis of Facial Images, IEEE Transactions on Pattern Analysis and Machine Intelligence, 28(5), pp.738-752 (2006).

4. Vision Data Recorder VD-100, http://www.dhands.co.jp/vd100/html/vd100.htm.

5. May, J.G., Kennedy, R.S., Williams, M.C., Dunlap, W.P. and Brannan J.R. (1990). Eye movement indices of mental workload, Acta Psychologica, Elsevier, 75(1), pp.75-89.

6. Haskell C.F., Kennedy D.O., Milne A.L., Wesnes K.A., Scholey A.B.; The effects of L-theanine, caffeine and their combination on cognition and mood, Biological Psychology, 77(2), pp.113-122 (2008).

7. Yoto A., Murao S., Motoki M., Yokoyama Y., Horie N., Takeshima K., Masuda K., Kim M., and Yokogoshi H.; Oral intake of $\gamma$-aminobutyric acid affects mood and activities of central nervous system during stressed condition induced by mental tasks, Amino Acids, Springer, pp.1-7 (2011).

8. Van der Pijl PC, Chen L., and Mulder TPJ; Human disposition of L-theanine in tea or aqueous solution, J. Funct Foods, 2, pp.239-244 (2010).

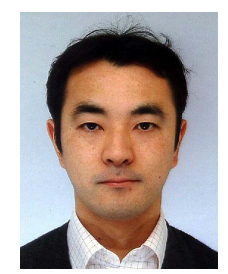

\section{Tsuyoshi MORIYAMA}

Tsuyoshi Moriyama is an assistant professor in the Department of Media and Image Technology, Tokyo Polytechnic University since 2007. After he received his Doctoral degree in Electrical Engineering from Keio University, Japan, in 1999, he joined the Institute of Industrial Science, University of Tokyo as a JSPS research fellow (2000-2001), the Robotics Institute, Carnegie Mellon University, U.S. as a postdoctoral fellow (2001-2004), and Dept. of Information and Computer Science, Keio University as an assistant professor (2004-2007). He has worked in many multidisciplinary projects, including analysis/synthesis of emotion in speech, automated analysis of facial expressions in images, automated summarization of movie films, software engineering, and design and development of multimedia contents. He is a member of the IEEE, IEICE, IPSJ, ASJ, and VRSJ. He received IEICE Young Investigators Award 1998.

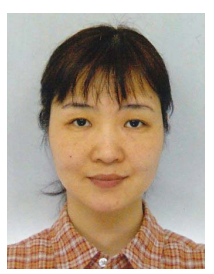

\section{Ai YOTO}

Ai Yoto is a researcher at School of Food and Nutritional Sciences, University of Shizuoka since 2007, after she earned the Ph.D. in Engineering from graduate school of engineering, Chiba University. She is engaged in researches on evaluating effects of food or food component intakes and effects of stimuli from other industrial products by physiological and psychological measurements. She is interested in researches about affective reaction, emotional stress and mental health currently.

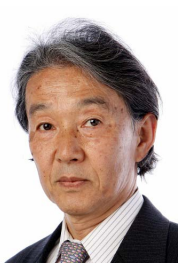

Hidehiko YOKOGOSHI

Hidehiko Yokogoshi, Doctor of Agriculture (Nagoya University, 1976), has been a professor at the Food and Nutritional Sciences Department, University of Shizuoka since 1993. He is an active researcher investigating the pharmacology and application of many amino acids in foods, such as L-theanine and gamma-aminobutyric acid (GABA) contained in green tea leaves and other foods. Being engaged in nutritional biochemistry and nutritional neuroscience, his research interests are nutritional metabolic specificities of amino acids and protein, behavioral science of food composition, neurotransmitter and brain function. The present position is a prof. of Chubu University, and an emeritus prof. of University of Shizuoka. 